\title{
Author index volume 17 (2015)
}

Abardia, J. \& Saorín Gómez, E., How do difference bodies in complex vector spaces look like? A geometrical approach

17 (2015) 1450023

Abdellaoui, B., Biroud, K., Davila, J. \& Mahmoudi, F., Nonlinear elliptic problem related to the Hardy inequality with singular term at the boundary

17 (2015) 1450033

Adamczak, R. \& Chafaï, D., Circular law for random matrices with unconditional logconcave distribution

Aizicovici, S., Papageorgiou, N. S. \& Staicu, V., Dirichlet problems with singular and superlinear terms

17 (2015) 1550056

Akagi, G. \& Kajikiya, R., Stability of stationary solutions for semilinear heat equations with concave nonlinearity

Alonso Álvarez, J. N., Fernández Vilaboa, J. M., González Rodríguez, R. \& Soneira Calvo, C., Cleft comodules over Hopf quasigroups

Alves, C. O., Carvalho, M. L. M. \& Gonçalves, J. V. A., On existence of solution of variational multivalued elliptic equations with critical growth via the Ekeland principle

17 (2015) 1550007
Azzollini, A., A note on the elliptic Kirchhoff equation in $\mathbb{R}^{N}$ perturbed by a local nonlinearity

17 (2015) 1450039

Badiale, M., Guida, M. \& Rolando, S., A nonexistence result for a nonlinear elliptic equation with singular and decaying potential

17 (2015) 1450024

Bantay, P., A trace formula for vector-valued modular forms

17 (2015) 1550069

Bétermin, L. \& Zhang, P., Minimization of energy per particle among Bravais lattices in $\mathbb{R}^{2}$ : Lennard-Jones and Thomas-Fermi cases

17 (2015) 1450049

Biagi, S. \& Bonfiglioli, A., A completeness result for time-dependent vector fields and applications

17 (2015) 1450040

Bidaut-Véron, M.-F. \& Nguyen, Q.-H., Evolution equations of $p$-Laplace type with absorption or source terms and measure data

17 (2015) 1550006

Biroud, K., see Abdellaoui, B.

17 (2015) 1450033

Bonfiglioli, A., see Biagi, S.

17 (2015) 1450040

Buchholz, A. \& Markwig, H., Tropical covers of curves and their moduli spaces

17 (2015) 1350045

Calamai, A. \& Spadini, M., Periodic perturbations of constrained 
motion problems on a class of implicitly defined manifolds

17 (2015) 1450027

Cao, X., see Shi, W.

Carvajal, X., Panthee, M. \& Scialom, M., On well-posedness of the third-order nonlinear Schrödinger equation with time-dependent coefficients

Carvalho, M. L. M., see Alves, C. O.

17 (2015) 1450031

17 (2015) 1450038

Catino, G., Mazzieri, L. \& Mongodi, S., Rigidity of gradient Einstein shrinkers

Ceballos, M., Núñez, J. \& Tenorio, Á. F., Abelian subalgebras on Lie algebras

Chafaï, D., see Adamczak, R.

Charro, F. \& Parini, E., On the existence threshold for positive solutions of $p$ Laplacian equations with a concave-convex nonlinearity

Cingolani, S., Vannella, G. \& Visetti, D., Multiplicity and nondegeneracy of positive solutions to quasilinear equations on compact Riemannian manifolds

Corrêa Jr, M., Maza, L. G. \& Soares, M. G., Hypersurfaces invariant by Pfaff systems

Correia, S., Blowup for the nonlinear Schrödinger equation with an inhomogeneous damping term in the $L^{2}$-critical case

17 (2015) 1450030

Dan, A., On a conjecture by Griffiths and Harris concerning certain Noether-Lefschetz loci

Davila, J., see Abdellaoui, B.

Dipierro, S. \& Pinamonti, A., Symmetry

17 (2015) 1550020

17 (2015) 1450044
17 (2015) 1550002

17 (2015) 1450033 results for stable and monotone solutions to fibered systems of PDEs

17 (2015) 1450035

Dubois, J. \& Wegner, C., Weighted $L^{2}$-invariants and applications to knot theory

17 (2015) 1450010

Fernández Vilaboa, J. M., see Alonso Álvarez, J. $\mathrm{N}$.

17 (2015) 1550007

Ferreira, L. C. F. \& Mesquita, C. A. A. S., An approach without using Hardy inequality for the linear heat equation with singular potential

17 (2015) 1550041

Fiorenza, A., Rakotoson, J. M. \& Sbordone, C., Variable exponents and grand Lebesgue spaces: Some optimal results

Fragnelli, G., Mugnai, D., Nistri, P. \& Papini, D., Nontrivial, nonnegative periodic solutions of a system of singulardegenerate parabolic equations with nonlocal terms

$\mathrm{Fu}$, Q., BLM realization for the integral form of quantum $\mathfrak{g l}_{n}$

Galdi, G. P., Metafune, G., Spina, C. \& Tacelli, C., Homogeneous CalderónZygmund estimates for a class of second-order elliptic operators

17 (2015) 1450017

Ganguly, D. \& Sandeep, K., Nondegeneracy of positive solutions of semilinear elliptic problems in the hyperbolic space

17 (2015) 1450019

Gasiński, L., O'Regan, D. \& Papageorgiou, N. S., A variational approach to nonlinear logistic equations

17 (2015) 1450021

Ge, J., Comparison theorems for manifolds with mean convex boundary
17 (2015) 1450025

17 (2015) 1550023

17 (2015) 1550019 
Gonçalves, J. V. A., see Alves, C. O.

17 (2015) 1450038

González Rodríguez, R., see Alonso Álvarez, J. $\mathrm{N}$.

17 (2015) 1550007

Goyal, S. \& Sreenadh, K., The Nehari manifold approach for $N$-Laplace equation with singular and exponential nonlinearities in $\mathbb{R}^{N}$

Guida, M., see Badiale, M.

Huang, Y., Wu, T.-F. \& Wu, Y., Multiple positive solutions for a class of concave-convex elliptic problems in $\mathbb{R}^{N}$ involving sign-changing weight, II

Jerrard, R. L., Novaga, M. \& Orlandi, G., On the regularity of timelike extremal surfaces

Kajikiya, R., see Akagi, G.

Kamin, S. \& Punzo, F., Prescribed conditions at infinity for parabolic equations

Kwong, K.-K. \& Miao, P., Monotone quantities involving a weighted $\sigma_{k}$ integral along inverse curvature flows

Li, C., Remarks on logarithmic K-stability

Llibre, J. \& Oliveira, R. D. S., Quadratic systems with invariant straight lines of total multiplicity two having Darboux invariants

Mahmoudi, F., see Abdellaoui, B.

Marino, V., Pacella, F. \& Sciunzi, B., Blow up of solutions of semilinear heat equations in general domains

Markwig, H., see Buchholz, A.

Maza, L. G., see Corrêa Jr, M.
17 (2015) 1550014

17 (2015) 1450020

17 (2015) 1350042

17 (2015) 1450011

17 (2015) 1450024

17 (2015) 1450045

17 (2015) 1450048

17 (2015) 1550001

17 (2015) 1450004

17 (2015) 1450018

17 (2015) 1450033

17 (2015) 1350045

17 (2015) 1450051
Mazurek, R. \& Ziembowski, M., On right McCoy rings and right McCoy rings relative to u.p.-monoids

17 (2015) 1550049

Mazzieri, L., see Catino, G.

17 (2015) 1550046

Mesquita, C. A. A. S., see Ferreira, L. C. F.

17 (2015) 1550041

Metafune, G., see Galdi, G. P.

Miao, C. \& Zheng, J., Scattering theory below energy space for two-dimensional nonlinear Schrödinger equation

17 (2015) 1450052

Miao, P., see Kwong, K.$\mathrm{K}$.

17 (2015) 1550014

Milman, V. \& Rotem, L., Characterizing addition of convex sets by polynomiality of volume and by the homothety operation

17 (2015) 1450022

Molchanov, I., Continued fractions built from convex sets and convex functions

17 (2015) 1550003

Molica Bisci, G. \& Rădulescu, V. D., Applications of local linking to nonlocal Neumann problems

17 (2015) 1450001

Mongodi, S., see Catino, G.

17 (2015) 1550046

Moroz, V. \& Van Schaftingen, J., Groundstates of nonlinear Choquard equations: HardyLittlewood-Sobolev critical exponent 17 (2015) 1550005

Mugnai, D., see Fragnelli, G.

17 (2015) 1450025

Neher, E., Pianzola, A., Prelat, D. \& Sepp, C., Invariant bilinear forms of algebras given by faithfully flat descent

Nguyen, Q.-H., see Bidaut-Véron, M.-F. Nistri, P., see Fragnelli, G.
$17(2015) 1450009$ 17 (2015) 1550006 17 (2015) 1450025 
Novaga, M., see Jerrard, R. L.

17 (2015) 1450048

Núñez, J., see Ceballos, M.

Oliveira, R. D. S., see Llibre, J.

O'Regan, D., see Gasiński, L.

17 (2015) 1550050

17 (2015) 1450018

17 (2015) 1450021

Orlandi, G., see Jerrard, R. L.

17 (2015) 1450048

Pacella, F., see Marino, V.

Panthee, M., see Carvajal, X.

17 (2015) 1350042

17 (2015) 1450031

Papageorgiou, N. S., see Aizicovici, S.

17 (2015) 1550056

Papageorgiou, N. S., see Gasiński, L.

17 (2015) 1450021

Papini, D., see Fragnelli, G.

17 (2015) 1450025

Parini, E., see Charro, F. 17 (2015) 1450044

Park, K.-S., Almost hsemi-slant Riemannian maps to almost quaternionic Hermitian manifolds

Pianzola, A., see Neher, E.

Pinamonti, A., see Dipierro, S.

Pisante, A. \& Punzo, F., Allen-Cahn approximation of mean curvature flow in Riemannian manifolds, II: Brakke's flows

Pourbarat, M., Stable intersection of middle$\alpha$ Cantor sets

17 (2015) 1550030

17 (2015) 1450009

17 (2015) 1450004

Punzo, F., see Kamin, S.

Punzo, F., see Pisante, A.

Qiu, G., A family of higher-order isoperimetric inequalities

Radnell, D., Schippers, E. \& Staubach, W., A Hilbert manifold structure on the Weil-Petersson class Teichmüller space of bordered Riemann surfaces

17 (2015) 1550016
Rădulescu, V. D., see Molica Bisci, G.

17 (2015) 1450001

Rakotoson, J. M., see Fiorenza, A.

17 (2015) 1550023

Ribeiro, B., Critical elliptic problems in $\mathbb{R}^{2}$ involving resonance in high-order eigenvalues

Rolando, S., see Badiale, M.

17 (2015) 1450008

17 (2015) 1450024

Rotem, L., see Milman, V.

Ruan, Z., Witt, I. \& Yin, H., On the existence and cusp singularity of solutions to semilinear generalized Tricomi equations with discontinuous initial data

17 (2015) 1450028

Sandeep, K., see Ganguly, D.

17 (2015) 1450019

Saorín Gómez, E., see Abardia, J.

17 (2015) 1450023

Sawon, J., On Lagrangian fibrations by Jacobians, II

17 (2015) 1450046

Sbordone, C., see Fiorenza, A.

17 (2015) 1550023

Schippers, E., see Radnell, D.

17 (2015) 1550016

Scialom, M., see Carvajal, $\mathrm{X}$.

17 (2015) 1450031

Sciunzi, B., see Marino, V.

17 (2015) 1350042

Sepp, C., see Neher, E.

Shelah, S., Dependent theories and the generic pair conjecture

Shi, W. \& Cao, X., Weyl's theorem for the square of operator and perturbations

17 (2015) 1450042

Soares, M. G., see Corrêa $\mathrm{Jr}, \mathrm{M}$.

17 (2015) 1450051

Soneira Calvo, C., see Alonso Álvarez, J. N.

Song, H. J. \& Yin, J. X., Singularities of solutions to the weighted $p$ Laplacian with an isolated singular point 17 (2015) 1450037 Spadini, M., see Calamai, A.

17 (2015) 1450027

Spina, C., see Galdi, G. $P$. 
Sreenadh, K., see Goyal, S.

17 (2015) 1450011

Staicu, V., see Aizicovici, S.

Staubach, W., see Radnell, D.

17 (2015) 1550056

17 (2015) 1550016

Su, Y. \& Yue, X., Classification of $\mathbb{Z}^{2}$-graded modules of intermediate series over a Blocktype Lie algebra

Tacelli, C., see Galdi, G. P.

17 (2015) 1550059

17 (2015) 1450017

Takahashi, F., Extremal solutions to LiouvilleGelfand type elliptic problems with nonlinear Neumann boundary conditions

17 (2015) 1450016

Tenorio, Á. F., see Ceballos, M.

Tesfahun, A., Almost critical local wellposedness for the spacetime monopole equation in Lorenz gauge

Van Schaftingen, J., see Moroz, V.

Vannella, G., see Cingolani, $\mathrm{S}$.

17 (2015) 1550050

17 (2015) 1450043

17 (2015) 1550005

17 (2015) 1450029

Visetti, D., see Cingolani, S.
Vitagliano, L., On the strong homotopy associative algebra of a foliation

Vrabie, I. I., Delay evolution equations with mixed nonlocal plus local initial conditions

Wang, Y., Xie, N. \& Zhang, X., The positive energy theorem for asymptotically anti-de Sitter spacetimes 17 (2015) 1550015

Wegner, C., see Dubois, J.

17 (2015) 1450010

Witt, I., see Ruan, Z. 17 (2015) 1450028

Wu, T.-F., see Huang, Y. 17 (2015) 1450045

Wu, Y., see Huang, Y. 17 (2015) 1450045

Xie, N., see Wang, Y. 17 (2015) 1550015

Yin, H., see Ruan, Z. 17 (2015) 1450028

Yin, J. X., see Song, H. J. 17 (2015) 1450037

$\mathrm{Yu}, \mathrm{X}$., Liouville type theorem for nonlinear elliptic equation involving Grushin operators $\mathbf{1 7}$ (2015) 1450050 Yue, X., see Su, Y. 17 (2015) 1550059 Zhang, P., see Bétermin, L.

17 (2015) 1450049

Zhang, X., see Wang, Y. 17 (2015) 1550015

Zheng, J., see Miao, C. 17 (2015) 1450052

Ziembowski, M., see Mazurek, R.

17 (2015) 1550049 